\title{
Numerical and Experimental Study of a Novel Concept for Hydraulically Controlled Negative Loads
}

\author{
Jesper Kirk Sørensen ${ }^{1}$ Michael Rygaard Hansen ${ }^{1}$ Morten Kjeld Ebbesen ${ }^{1}$ \\ Department of Engineering Sciences, Faculty of Engineering and Science, University of Agder Jon Lilletunsvei 9 , \\ 4879 Grimstad, Norway. E-mail: jesper.k.sorensen@uia.no, michael.r.hansen@uia.no, morten.k.ebbesen@uia.no
}

\begin{abstract}
This paper presents a numerical and experimental investigation of a novel concept that eliminates oscillations in hydraulic systems containing a counterbalance valve in series with a pressure compensated flow supply. The concept utilizes a secondary circuit where a low-pass filtered value of the load pressure is generated and fed back to the compensator of the flow supply valve. The novel concept has been implemented on a single boom actuated by a cylinder. A nonlinear model of the system has been developed and an experimental verification shows good correspondence between the model and the real system. The model is used for a parameter study on the novel concept. From the study it is found that the system is stable for large directional valve openings and that for small openings a reduction of the oscillatory behaviour of the system can be obtained by either lowering the eigenfrequency of the mechanical-hydraulic system or by lowering the pilot area ratio of the counterbalance valve.
\end{abstract}

Keywords: counterbalance valve, pressure compensated valve, instabilities in hydraulic systems, nonlinear model, load-holding application

\section{Introduction}

For safety reasons, hydraulic load carrying applications are required by law to contain a load holding protection device. The most widely used device is the counterbalance valve $(\mathrm{CBV})$. It is multi-functional and provides leak tight load holding, load holding at hose/pump failure as well as shock absorption, overload protection, and cavitation prevention at load lowering. However, it is well known that a series connection of a pressure compensator (CV), a directional control valve (DCV), and a CBV tends to introduce instability in a system, see Miyakawa (1978), Persson et al. (1989), Handroos et al. (1993), Zähe (1995) and Hansen and Andersen (2010). This is mainly a problem when the controlled actuator is subjected to a negative load, i.e., a load that tends to drive the actuator as a pump, because this will require the counterbalance valve to throttle the return flow, see Figure 1. The system in Figure 1 will be referred to as the base circuit.

It is a major challenge within hydraulic system design to find solutions that offer stable handling of negative loads together with pressure compensated metering-in flow. Typically, practical solutions will compromise either the load independency, the response time or the level of oscillations (Hansen and Andersen (2001) and Nordhammer et al. (2012)). The consequences of the oscillatory nature of such systems are reduced safety, reduced productivity as well as added fatigue load on both the mechanical and hydraulic system. The severity of oscillations is affected by a wide variety of parameters some of which are hard to predict or change: the external load on the actuator, the properties of the mechanical structure, the friction in 


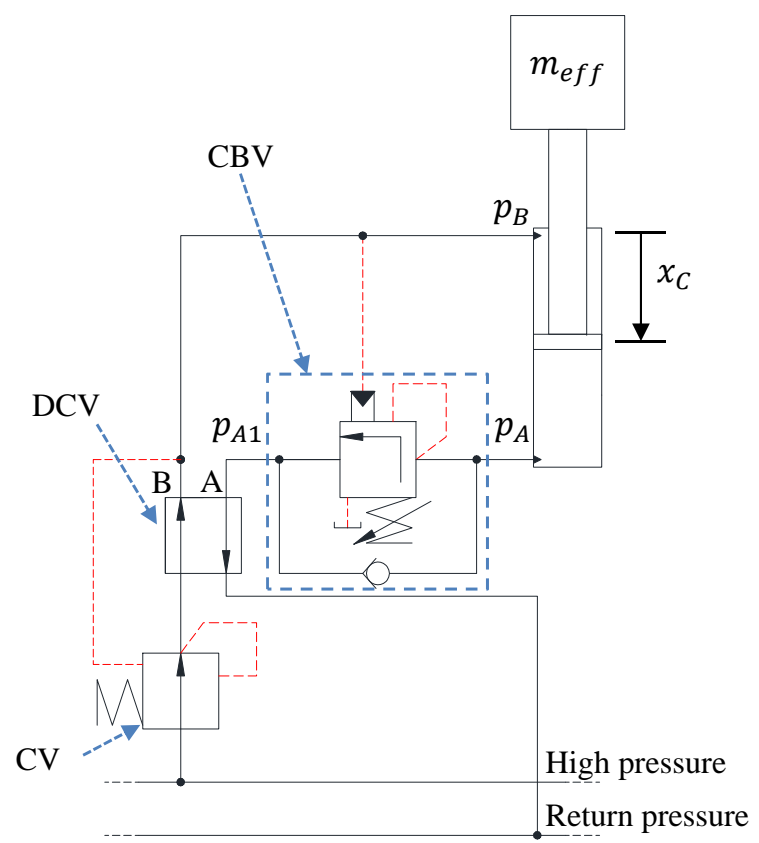

Figure 1: The base circuit consisting of a pressure compensator $(\mathrm{CV})$, a directional control valve (DCV), a counterbalance valve (CBV), and a cylinder subjected to a compressing load.

the cylinder, the damping and hysteresis of the CBV, the operator input as well as volumes and restrictions in the hydraulic lines. The efforts to minimize the oscillatory nature of the base circuit can be divided into three groups: Parameters variations (pilot area ratio of $\mathrm{CBV}$, pilot line orifices, etc.) on the circuit using the same main components. The parameters with most influence on the stability are the damping of the system and the pilot ratio of the CBV (Hansen and Andersen, 2001). However lowering the pilot ratio of the CBV to minimize the oscillations will increase pressure levels and hence increase power consumption - especially with small external loads. Another approach is to add damping when designing the pilot line leading in to the CBV. However, no unique solution has emerged that is useful across applications and working conditions. A different approach is to actively compensate for the oscillations by applying closed-loop control strategies that involve the input signal to the DCV and some kind of pressure feedback (Hansen and Andersen (2010), Cristofori et al. (2012) and Ritelli and Vacca (2014)). The most important limitation in these strategies is the bandwidth limitation in typical DCVs. Alternatively, the pressure compensator (CV) can be removed and the DCV replaced by a servo valve which is a proven and reliable method for motion control. The weaknesses here are in the investment costs and the difficulties in handling disturbances in the supply pressure caused by neighbouring circuits. The authors have investigated the use of a DCV with compensated supply pressure, see Sørensen et al. (2014) and Sørensen et al. (2015). This is a commercially available alternative that is characterized by low cost but also load dependent flow. Another example is described in Nordhammer et al. (2012), where the main throttling ability is moved from the CBV to the return orifice of the DCV, thereby eliminating the oscillations. However, this is not a viable solution if the minimum load is $60 \%$ or less of the maximum load, which strongly minimizes the applicability. All of the approaches have certain drawbacks as compared to the base circuit. The authors have previously presented a novel concept for addressing this stability issue (Sørensen et al., 2016). It has the same steady state flow characteristics as the base circuit only without the corresponding oscillatory nature. The concept was implemented on an experimental setup and its ability to suppress oscillations was experimentally verified. This paper is devoted to the nonlinear modelling of the new concept with a view to investigate and predict the performance of the concept with special emphasis on stability.

\section{Novel concept}

In Figure 2 a hydraulic diagram of the proposed concept is shown, patent pending (Hansen and Sørensen, 2015). It is shown in a situation where the actuator is subjected to a negative load, i.e., a lowering motion of some gravitational payload.

When compared to the base circuit in Figure 1, it can be seen that the pilot pressure connection of the $\mathrm{CV}$ is supplied by the secondary circuit rather than by the B-port pressure. The underlying idea is to suppress oscillations in the system by generating the steady state value of the B-port pressure in the secondary circuit, filtering out any oscillations. The concept also encompasses solutions where the secondary circuit is connected to the $\mathrm{CBV}$ or both the $\mathrm{CV}$ and the CBV. The version used in this paper where only the $\mathrm{CV}$ is connected to the secondary circuit, is however the preferred one from a reliability point of view. This is because the CBV and the related safety functions are activated independent of any electrical system. Since the concept is passive as seen from the operator's point of view it can be combined with any closed loop control strategies on the DCV. In this paper, the concept presented is with a linear actuator, but the method will also work for rotational actuators in circuits with counterbalance valves.

The concept employs an orifice and a proportional pressure relief valve $(\mathrm{PV})$ in series. The intermediate pressure, $p_{C}$, is connected to the $\mathrm{CV}$ and will be 


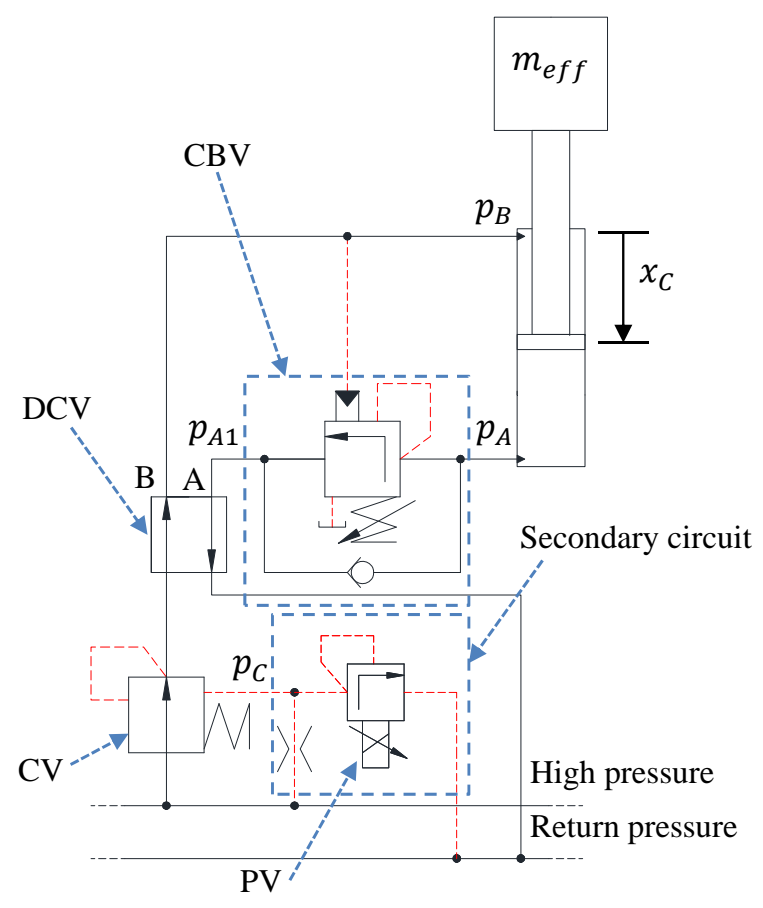

Figure 2: Hydraulic diagram of novel concept where the $\mathrm{CV}$ is connected to the secondary circuit.

referred to as the compensator pressure. The overall target is that $p_{C}$ shall be the steady state value of $p_{B}$ thereby suppressing oscillations of the compensator and, subsequently, in the entire system. For that purpose, a control strategy is suggested that requires the measurement of $p_{B}$, a low-pass filtering yielding a reference value for the compensator pressure, $p_{C}^{r e f}$ and a measurement of pressure, $p_{C}$. This allows for a closed loop control where the pressure setting of the proportional pressure relief valve is adjusted by means of a control signal, $u_{P V}$, in order to continuously meet the reference value of the compensator pressure. A block diagram of the used control strategy is shown in Figure 3 .

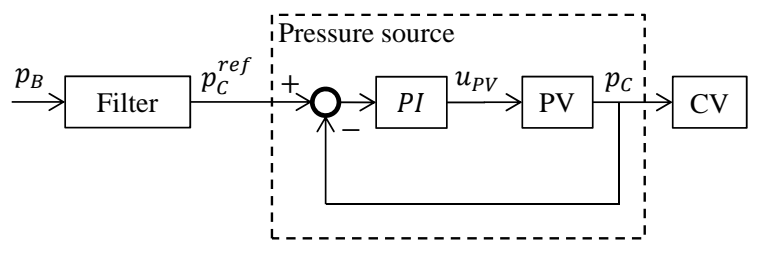

Figure 3: The proposed control strategy.

Figure 4 demonstrates the effect of the concept. It compares the pressures on both sides of the cylinder for the base circuit and the concept when providing a ramp input downwards to a simple load-carrying boom, see further details in section 3 . The base circuit is unstable and the ability of the concept to suppress oscillations in a real system is clear.

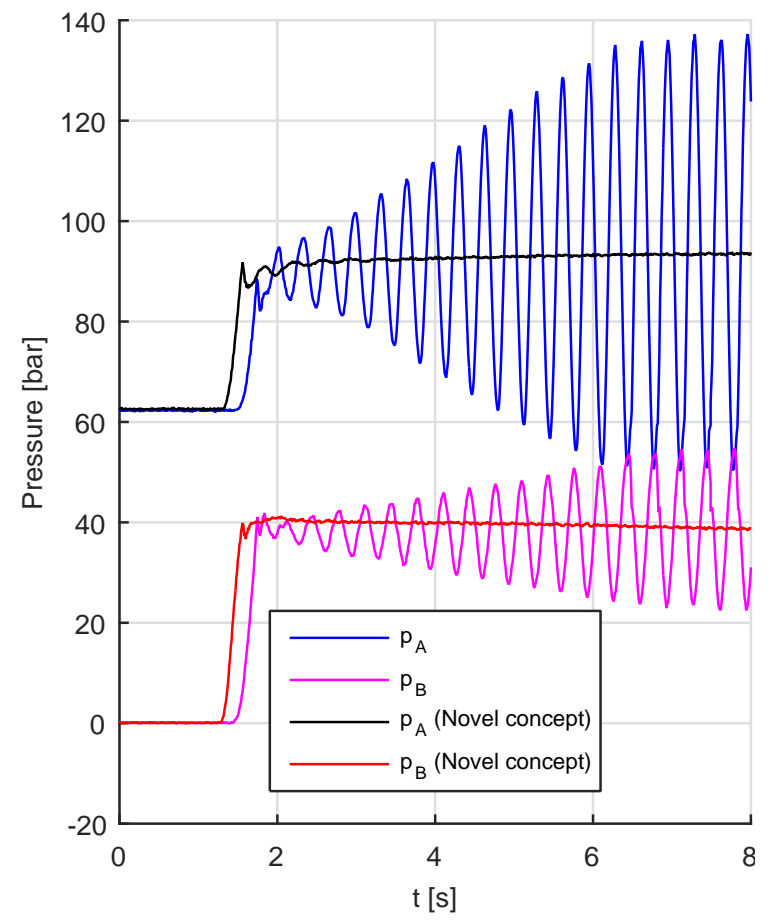

Figure 4: Comparison of pressures between the base circuit and the system with the novel concept implemented for a DCV ramp input.

\section{Considered system}

In order to examine the concept in more detail investigations have been conducted on a setup in the mechatronics laboratory at the University of Agder, see Figure 5. The setup consists of a hydraulically actuated boom and a control system.

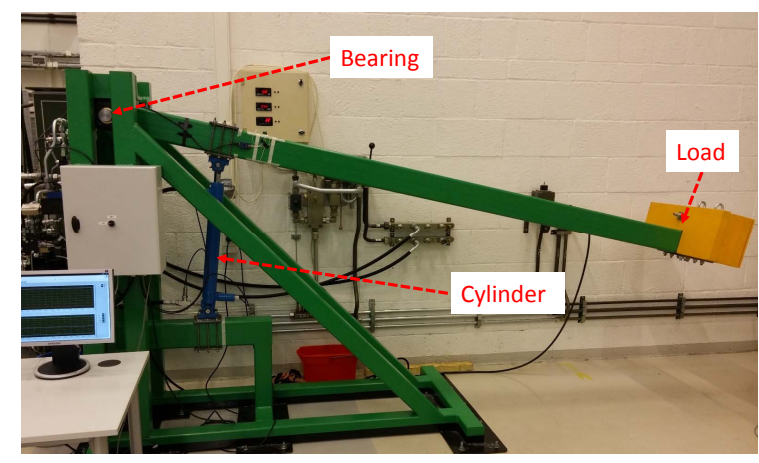

Figure 5: Hydraulic boom experimental setup.

The hydraulics can easily be altered from the novel concept in Figure 2 to the base circuit in Figure 1. 
The concept has been implemented using commercially available components. The DCV and CV are embedded in a pressure compensated 4/3-way directional control valve group from Danfoss (Model: PVG32). It has an electrohydraulic actuation with linear flow vs. input signal characteristics with a maximum value of $Q_{D C V}^{\max }=25 \mathrm{~L} / \mathrm{min}$. The 4 -port $\mathrm{CBV}$ is from Sun Hydraulics (Model: CWCA) with a $3: 1$ pilot area ratio and a rated flow of $Q_{C B V}^{r}=60 L /$ min. The PV is from Bosch Rexroth (Model: DBETE) and has a crack pressure that varies linearly with the voltage input. At maximum signal, $u_{P V}^{\max }=1$, the valve cracks open at $p_{C @ 0 L / \min }=185 \mathrm{bar}$ and has a rated pressure $p_{P V}^{r}=p_{C @ 0.8 L / \text { min }}=200$ bar. In Table 1 are listed some other design parameters of the experimental setup.

Table 1: Design parameters of the experimental setup.

\begin{tabular}{lc}
\hline Parameter & Value \\
\hline Distance from bearing to & $L=3570 \mathrm{~mm}$ \\
mass centre of boom + load. & $m=410 \mathrm{~kg}$ \\
Mass of boom + load & $H_{c}=500 \mathrm{~mm}$ \\
Cylinder stroke & $D_{p}=65 \mathrm{~mm}$ \\
Cylinder piston diameter & $D_{r}=35 \mathrm{~mm}$ \\
Cylinder rod diameter & $\mu_{C}=\frac{D_{p}^{2}}{D_{p}^{2}-D_{r}^{2}}=1.41$ \\
Cylinder area ratio & $p_{S}=180 \mathrm{bar}$ \\
Supply pressure &
\end{tabular}

A real-time I/O system is used to control the hydraulic valves on the boom with a loop time of $10 \mathrm{~ms}$. The control system can record sensor information from all the position and pressure sensors mounted on the test setup. The primary circuit is activated by supplying the directional control valve with an input signal. The purpose of the controller on the secondary circuit is to keep the compensator pressure, $p_{C}$, in accordance with the reference pressure $p_{C}^{r e f}$, in Figure 3 . The filter box uses the actual $p_{B}$ value as input and returns $p_{C}^{r e f}$. The choice of filter frequency should of course reflect both the dominant lowest eigenfrequency of the mechanical-hydraulic system as well as the demand for a certain response time of the system. The role of the low-pass filter is to remove oscillations, however if it is chosen overly conservative then the system reacts too slowly. Therefore, some logic has been added so that the compensator reference pressure, $p_{C}^{r e f}$, never goes below a certain minimum value, $p^{\text {min }}$ :

$$
p_{C}^{r e f}= \begin{cases}p^{\text {min }} & , p_{B}<p^{\text {min }} \\ p_{B, L P F} & , p_{B} \geq p^{\text {min }}\end{cases}
$$

where $p_{B, L P F}$ is the low-pass filtered value of $p_{B}$ :

$$
\dot{p}_{B, L P F}=\frac{1}{\tau} \cdot\left(p_{B}-p_{B, L P F}\right)
$$

The PI-controller has the classic form:

$$
u_{P V}=K_{P} \cdot\left(p_{C}^{r e f}-p_{C}\right)+\int K_{I} \cdot\left(p_{C}^{r e f}-p_{C}\right) \cdot d t
$$

where saturation and corresponding anti-windup measures (integrated effort not accumulated at saturation) are implemented so that $0 \leq u_{P V} \leq 1$. Basically, only four parameters need to be set: $p^{\text {min }}, \tau, K_{P}$ and $K_{I}$.

\section{Nonlinear model}

A nonlinear model of the system, both with and without the concept implemented, is developed using the commercial simulation software SimulationX. This section describes the theory behind the different parts of this model.

\subsection{Mechanical system}

The mechanical system used in the investigation of the concept for stabilizing the hydraulic circuit comprises a boom, a payload, a base, and a double acting hydraulic actuator - see Figure 6.

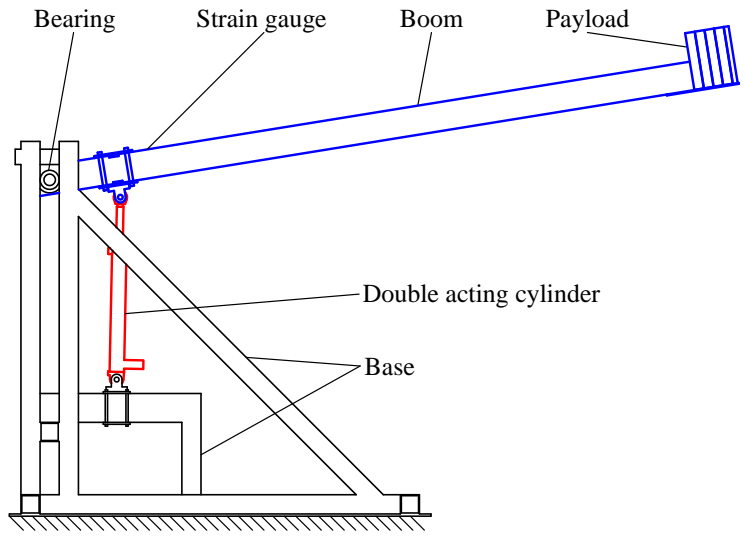

Figure 6: Mechanical system.

In the time domain simulation of the system the boom is modelled to be flexible using the finite segment method as described by Huston and Wang (1994). The method is now well tested for modelling the dynamic behaviour of flexible beam systems in a relatively simple way. In the finite segment method a beam is replaced with a number of smaller beam segments connected to each other with extension and/or torsional 
springs. With this method it is possible to model both bending, extension, and torsion of a beam. The system at hand is considered to be a planar mechanism and only bending is taken into account. The flexibility in the longitudinal direction of the beam is omitted as its influence on the dynamic behaviour of the system can be neglected. Therefore the segments in the present model are connected by revolute joints and torsional springs. Due to the segmented nature of the model it does not describe the deformed shape of the beam smoothly but this is not required for the problem at hand where the key point of interest is system oscillations. The segmented structure is illustrated in Figure 7.
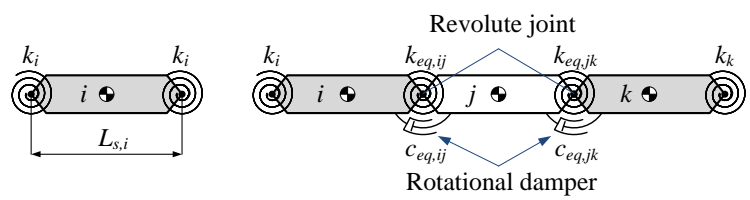

Figure 7: Illustration of torsional springs and dampers between segments in the finite segment method.

The torsional spring between two segments has the stiffness of two springs mounted in series. The stiffness $k_{i}$ of the spring related to segment number $i$ can be written as:

$$
k_{i}=\frac{2 \cdot E \cdot I_{z}}{L_{s, i}}
$$

where $E$ is the bulk modulus of the beam material, $I_{z}$ is the $2^{\text {nd }}$ moment of inertia for the cross section of the prismatic beam, and $L_{s, i}$ is the length of the segment. The equivalent spring stiffness $k_{e q, i j}$ between two segments can then be written as two springs in series:

$$
k_{e q, i j}=\frac{k_{i} \cdot k_{j}}{k_{i}+k_{j}}
$$

The number of segments in the model is a compromise between accuracy and computational time. To obtain a sufficiently good approximation of the eigenfrequency of the boom the model contains four segments between the bearing and cylinder and five segments between the cylinder and the payload. The base is considered to be rigid even though observations during the experimental work have revealed that the base also contributes to the flexibility in the system. To accommodate the flexibility of the base a tuning factor has been applied to the stiffnesses of the segments to tune the dynamic behaviour to experimental data.
The payload is considered to be a rigid point mass. As illustrated in Figure 7 a rotational damper is also included in the connection between two segments. The value of the damping coefficient, $c_{e q}$, is found through tuning to the experimental data.

\subsection{Hydraulic system}

The description of the hydraulic system is only developed for downwards motion of the boom.

\subsubsection{Directional control valve}

The directional control valve unit consists of a directional control valve in series with a pressure compensator valve. The valve has been modelled as two variable orifices as shown in Figure 8. The opening of these are controlled by a set of function blocks, including valve dynamics.

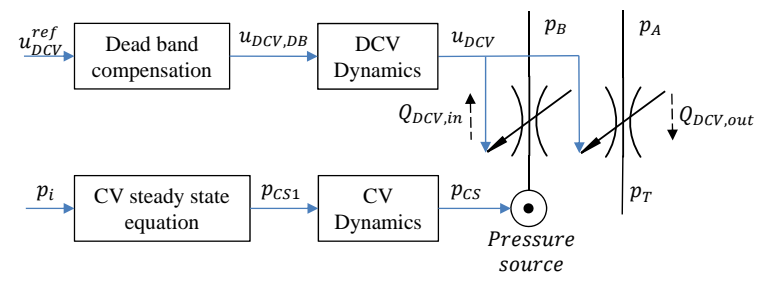

Figure 8: Structure of DCV model.

The blue lines are signal lines and the black lines are hydraulic lines. Assuming constant density and using the orifice flow equation then the flow across the DCV can be computed as:

$$
\begin{aligned}
Q_{D C V, \text { in }} & =k_{C S-B} \cdot u_{D C V} \cdot \sqrt{p_{C S}-p_{B}} \\
Q_{D C V, \text { out }} & =k_{A-T} \cdot u_{D C V} \cdot \sqrt{p_{A}-p_{T}}
\end{aligned}
$$

where $Q_{D C V \text {,in }}$ and $Q_{D C V \text {,out }}$ are the compensated metering-in flow and metering-out flow, $p_{T}$ is the tank pressure, $u_{D C V}$ is the dimensionless opening of the valve. The parameters $k_{C S-B}$ and $k_{A-T}$ are valve constants. The compensated supply pressure, $p_{C S}$, is calculated by the compensator equation, which is implemented like:

$$
p_{C S 1}= \begin{cases}p_{S} & , p_{i} \geq p_{S}-p_{D C V, c l} \\ p_{i}+p_{D C V, c l} & , p_{i}<p_{S}-p_{D C V, c l}\end{cases}
$$

where $p_{i}$ is the input pressure to the $\mathrm{CV}, p_{S}$ is the supply pressure and $p_{D C V, c l}$ is the nominal pressure drop across the main spool (setting of CV spring). The dynamics of the $\mathrm{CV}$ is added to account for the valve 
not being a perfect flow source. It is described by a first order transfer function:

$$
\frac{p_{C S}}{p_{C S 1}}(s)=\frac{1}{\tau_{C V} \cdot s+1}
$$

A difference between the model of the base circuit and the concept is the input pressure, $p_{i}$, to the CV:

$$
p_{i}= \begin{cases}p_{B} & , \text { for Base Circuit } \\ p_{C} & , \text { for Novel Concept }\end{cases}
$$

Experiments showed a slightly higher flow output of the DCV utilising the concept than of the base circuit. This indicates that equation (10) in reality looks like:

$$
p_{i}=\left\{\begin{array}{l}
p_{B}-\Delta p_{L S}^{B C}\left(u_{D C V}\right) \\
p_{C}-\Delta p_{L S}^{N C}\left(u_{D C V}\right)
\end{array}\right.
$$

where $\Delta p_{L S}$ is the pressure drop internally in the DCV's load sensing system before the CV which is a function of $u_{D C V}$. The difference between the two systems occurs because pressure $p_{C}$ is obtained by connecting the secondary circuit to an external port on the DCV, while pressure $p_{B}$ is handled internally in the DCV. The experiments indicate that $\Delta p_{L S}^{B C}>\Delta p_{L S}^{N C}$. The pressure drop $\Delta p_{L S}$, is combined with $k_{C S-B}$ in an equivalent valve characteristic $L_{C S-B}$, changing equation (6) to:

$$
\begin{aligned}
& Q_{D C V, i n}^{B C}=L_{C S-B}^{B C}\left(u_{D C V}\right) \cdot \sqrt{p_{C S}-p_{B}} \\
& Q_{D C V, i n}^{N C}=L_{C S-B}^{N C}\left(u_{D C V}\right) \cdot \sqrt{p_{C S}-p_{B}}
\end{aligned}
$$

In Figure 9 is shown $L_{C S-B}$ for both systems as a function of $u_{D C V}$.

The spool is open-centre, hence, it has no dead band on the outlet. For the inlet a dead band compensation is implemented as follows, where $\sigma_{D B}$ is the dimensionless dead band:

$$
u_{D C V, D B}= \begin{cases}0 & , u_{D C V}^{r e f}=0 \\ \sigma_{D B}+\left(1-\sigma_{D B}\right) \cdot u_{D C V}^{r e f} & , u_{D C V}^{r e f}>0\end{cases}
$$

The dynamics of the DCV is implemented using a second order transfer function:

$$
\frac{u_{D C V}}{u_{D C V, D B}}(s)=\frac{1}{\frac{s^{2}}{\omega_{D C V}^{2}}+2 \cdot \zeta_{D C V} \cdot \frac{s}{\omega_{D C V}}+1}
$$

where $\omega_{D C V}$ is the natural eigenfrequency of the valve and $\zeta_{D C V}$ is the damping ratio. Values for the parameters used in the modelling work can be found in Table 2.

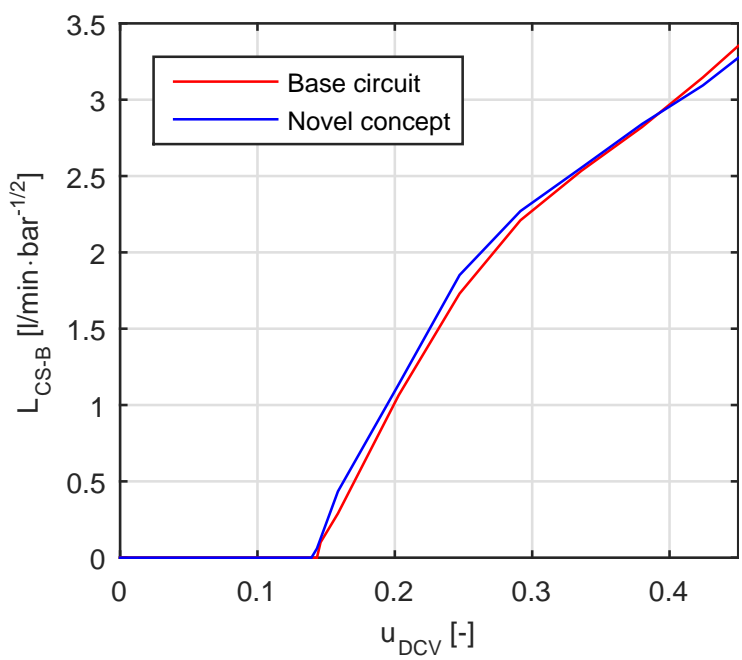

Figure 9: Equivalent valve characteristic, $L_{C S-B}$, as function of $u_{D C V}$.

Table 2: DCV model parameters.

\begin{tabular}{lc}
\hline Parameter & Value \\
\hline$k_{A-T}$ & $2.3 \frac{\mathrm{L}}{\min \sqrt{b a r}}$ \\
$p_{D C V, c l}$ & 7 bar $^{a}$ \\
$\tau_{C V}$ & $0.0064 s$ \\
$\sigma_{D B}$ & 0.14 \\
$\omega_{D C V}$ & $30 \frac{\mathrm{rad}}{\mathrm{s}}(4.8 \mathrm{~Hz})$ \\
$\zeta_{D C V}$ & $0.8^{a}$ \\
\hline
\end{tabular}

${ }^{a}$ Bak and Hansen (2013) 


\subsubsection{Counterbalance valve}

The valve is a 4-port vented valve from Sun Hydraulics. The counterbalance valve consists of two parts: a check valve and a pilot operated relief valve as shown in Figure 1 and Figure 2. Only the relief valve is modelled by means of a variable orifice as shown in Figure 10. The opening is controlled by a set of function blocks, including valve dynamics. The blue lines are signal lines and the black lines are hydraulic lines.

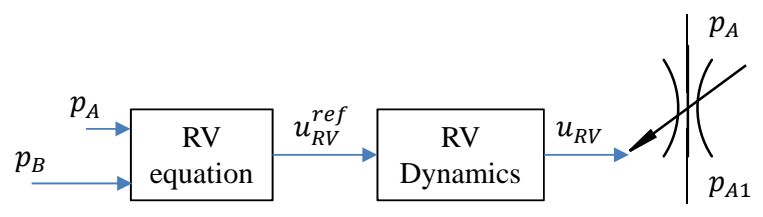

Figure 10: Structure of CBV model.

The flow, $Q_{C B V}$, through the valve is defined by the orifice equation:

$$
Q_{C B V}=k_{v, C B V} \cdot u_{R V} \cdot \sqrt{p_{A}-p_{A 1}}
$$

where $k_{v, C B V}$ is the counterbalance valve constant, and $u_{C B V}$ is the dimensionless opening of the relief valve. Assuming no back pressure from tank, the normalised reference opening, $0 \leq u_{R V}^{r e f} \leq 1$, is defined as:

$$
u_{R V}^{r e f}=\frac{\alpha_{P} \cdot p_{B}+p_{A}-p_{c r, R V}}{\Delta p_{o p, R V}}
$$

where $\alpha_{P}$ is the CBV pilot area ratio, $p_{c r, R V}$ is the crack pressure of the relief valve and $\Delta p_{o p}, R V$ is the extra pressure required to open the CBV fully. The dynamics of the relief valve poppet is described by a first order transfer function:

$$
\frac{u_{R V}}{u_{R V}^{r e f}}(s)=\frac{1}{\tau_{R V} \cdot s+1}
$$

where $\tau_{R V}$ is the time constant. Values for the CBV parameters can be seen in Table 3 .

\subsubsection{Hydraulic cylinder}

The model of the hydraulic cylinder is based on the one presented by Bak and Hansen (2013). The cylinder force, $F_{c y l}$, is defined as:

$$
F_{c y l}=F_{P}-\tanh \left(v_{C} \cdot C_{t h}\right) \cdot F_{f r}
$$

Table 3: CBV model parameters.

\begin{tabular}{lc}
\hline Parameter & Value \\
\hline$k_{v, C B V}$ & $1.90 \frac{L}{\min \sqrt{b a r}}$ \\
$p_{c r, R V}$ & $196 b a r$ \\
$\Delta p_{o p}, R V$ & $350 b a r$ \\
$\alpha_{P}$ & 3 \\
$\tau_{R V}$ & $0.089 \mathrm{~s}$ \\
\hline
\end{tabular}

It consists of the pressure induced force, $F_{P}$, and a friction component, $F_{f r}$. The hyperbolic tangent function is used to avoid numerical difficulties at zero velocity, however, the shape factor $C_{t h}$ is chosen sufficiently high to ensure that stiction between the piston and the cylinder can be simulated from negligible velocity fluctuation. The pressure induced force is defined as:

$$
F_{P}=p_{A} \cdot \mu_{C} \cdot A_{B}-p_{B} \cdot A_{B}
$$

The friction force is defined as:

$$
F_{f r}=F_{S}+C_{P} \cdot\left|F_{P}\right|
$$

where $F_{S}$ describes the force required to overcome the static friction and $C_{p}$ is a scaling factor for the pressure dependent friction. The friction parameters for the cylinder can be found in Table 4 .

Table 4: Hydraulic cylinder model parameters.

\begin{tabular}{lc}
\hline Parameter & Value \\
\hline$C_{t h}$ & $10300 \frac{s}{m}$ \\
$F_{S}$ & $\mu_{C} \cdot A_{B} \cdot 1 \cdot 10^{5} m^{2} P a$ \\
$C_{p}$ & 0.02 \\
\hline
\end{tabular}

\subsubsection{Secondary circuit}

The performance of the chosen implementation of the secondary circuit depends to a large extent on the used PV. However, experiments have shown that the internal leakage cannot be neglected, whether it is across the CV or other places in the load sensing system of the valve group. The leakage is modelled as a fixed orifice and the circuit is shown in Figure 11.

The orifice parameters are estimated from experiments, and they are listed in Table 5. The PV has its own factory set closed loop control aiming at linearising the flow-pressure characteristics. Based on measurements the following model for the flow has been identified. 


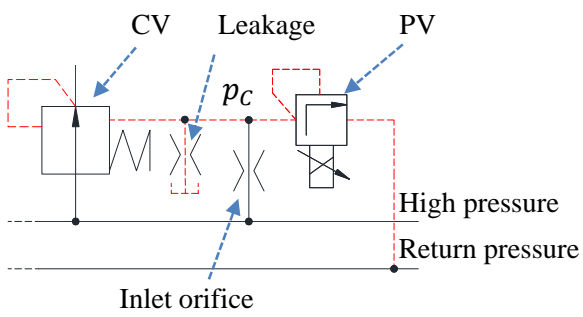

Figure 11: Actual secondary circuit including leakage in the load sensing system of the proportional valve group.

$$
Q_{P V}=k_{P V} \cdot \sigma_{P V} \cdot \sqrt{p_{C}}
$$

where $k_{P V}$ is the PV constant and $\sigma_{P V}$ is the dimensionless opening of the orifice internally in the PV. It is linked to the dimensionless input reference, $u_{P V}$, by the following equation:

$$
\sigma_{P V}= \begin{cases}\frac{p_{C}-u_{P V} \cdot p_{P V}^{r}}{\Delta p_{o p}, P V^{r}} & , u_{P V}>\sigma_{1} \\ \frac{p_{C}-u_{P V} \cdot p_{P V}}{\Delta p_{o p}, P V} \cdot \frac{\theta_{1}}{\left(u_{P V}\right)^{2}} & , \sigma_{0}<u_{P V}<\sigma_{1} \\ 1 & , u_{P V}=\sigma_{0}\end{cases}
$$

where $\sigma_{0}$ and $\sigma_{1}$ select the intervals of the piecewise function. $\theta_{1}$ is a constant to adjust the curvature. The range of pressure, $p_{C}$, available from the secondary circuit goes from $p_{C}^{\min }=3 \mathrm{bar}$ to $p_{C}^{\max }=75 \mathrm{bar}$.

Table 5: Secondary circuit model parameters

\begin{tabular}{lc}
\hline Parameter & Value \\
\hline Inlet orifice, pressure drop & $\left.\Delta p\right|_{@ 2 L / \text { min }}=220$ bar \\
at a flow $Q=2 \frac{L}{\min }$ & \\
Leakage, pressure drop at & $\left.\Delta p\right|_{@ 2 L / \text { min }}=160$ bar \\
a flow $Q=2 \frac{L}{\min }$ & $0.791 \frac{L}{\min \sqrt{b a r}}$ \\
$k_{P V}$ & $40 b a r$ \\
$\Delta p_{o p, P V}$ & $200 b a r$ \\
$p_{P V}^{r}$ & 0.0081 \\
$\theta_{1}$ & 0 \\
$\sigma_{0}$ & 0.09 \\
$\sigma_{1}$ & $3 b a r$ \\
$p_{C}^{\text {min }}$, PV fully open & $75 b a r$ \\
$p_{C}^{\text {max }}$, PV closed & \\
\hline
\end{tabular}

\section{Experimental verification of nonlinear model}

In this section the nonlinear model is verified against experimental results. First the behaviour of the parts of the system are verified before the model for the total system is. In general, when tuning/verifying the nonlinear model, it is being evaluated for four parameters: General tendency of the curves, peak sizes, the frequencies of the oscillations and finally the steady state values.

In this section, the following abbreviations are used:

Sim = Data from the nonlinear simulation model.

Exp $=$ Data from experiments.

\subsection{Cylinder friction}

Figure 12 shows the cylinder load as a function of piston position, with and without friction in the cylinder both from simulation and experiments (the curve without friction is calculated from the up and down curves). When moving the boom downwards the friction causes the experienced cylinder force of the cylinder to drop with the magnitude of the friction force and vice versa when moving upwards.

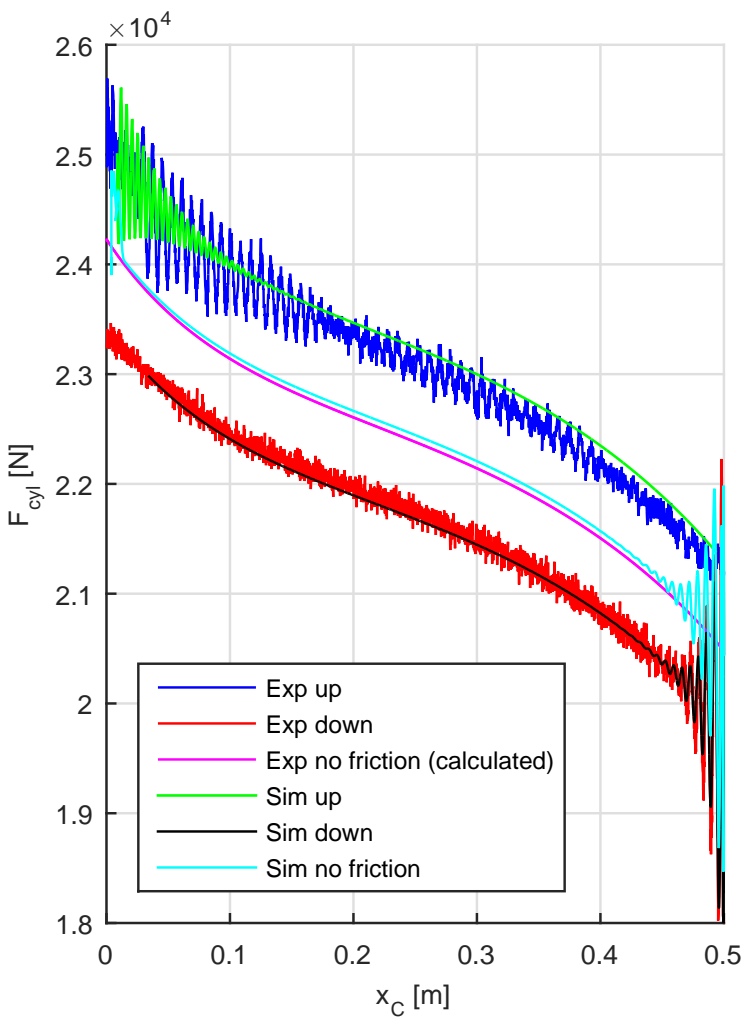

Figure 12: Load curves with and without friction.

The curves in Figure 12 are obtained by doing a full up-down cycle with constant cylinder velocity. The method used to model the cylinder seems to be a good fit in this case, since all three curves (up, down, and no 
friction) show a similar pattern as the measured ones. This also shows that the mechanical loads in the model are a good approximation of the real system.

\subsection{Eigenfrequency}

The pure mechanical eigenfrequency, $f_{m}$, and damping are found in the top and bottom position of the boom. In both of these experiments the piston is preloaded so that it is mechanically fixed to the relevant cylinder end plate. Next, the boom is excited manually and the motion is recorded. In Figure 13 the oscillations from the experiments are compared to the ones achieved from the simulation. From the experimental setup, strain is measured in the boom, see Figure 6, and from the simulation, the deflection of the nearby spring is used. The two data sets have been normalised to have the same amplitude at time $t=0 \mathrm{~s}$, see Figure 13. The comparison of strain and deflection respectively is considered to be acceptable.

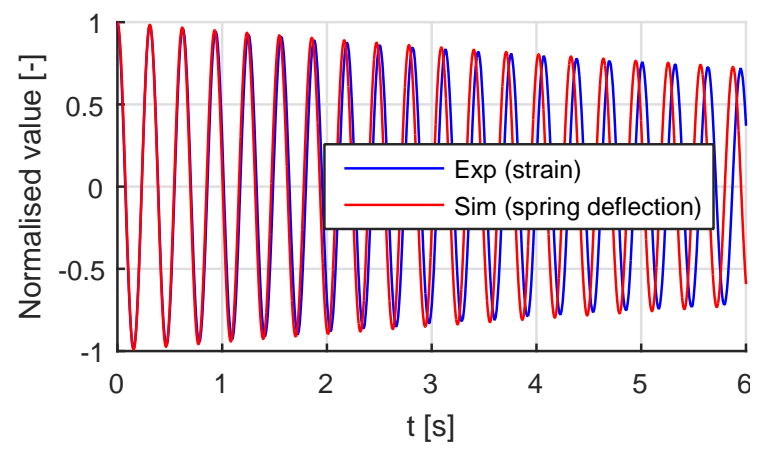

Figure 13: Normalised values of oscillations in top position $\left(x_{C}=0 m\right)$.

The curves show a good correspondence between simulation and experiments. The model has a slightly higher eigenfrequency. Due to the fact that the base is not included directly as a flexible part in the modelling of the mechanical structure but only as a tuning factor, the mechanical eigenfrequency from the model does not match the experiments perfectly over the entire span of operation. However, the difference is acceptable, see Table 6. The curves in Figure 13 also show that the mechanical damping is in accordance.

Table 6: Mechanical eigenfrequencies, $f_{m}$.

\begin{tabular}{lcc}
\hline Position & $f_{m}(\mathrm{Sim})$ & $f_{m}(\operatorname{Exp})$ \\
\hline Top & $3.2 \mathrm{~Hz}$ & $3.2 \mathrm{~Hz}$ \\
Bottom & $3.1 \mathrm{~Hz}$ & $3.2 \mathrm{~Hz}$ \\
\hline
\end{tabular}

The next step is to look at the combined mechanicalhydraulic eigenfrequency, $f_{m h}$, when the piston is sus- pended by two oil column springs in parallel. For that purpose investigations are carried out for two characteristic piston positions, $x_{C}=0.10 \mathrm{~m}$ and $x_{C}=0.25 \mathrm{~m}$, respectively. Figure 14 (upper) shows the normalised values of oscillations when the boom is placed with the piston at $x_{C}=0.10 \mathrm{~m}$ and a similar external force as before is applied to verify the spring effect of the hydraulic system. As it can be seen, the oscillations show a nice fit, including the damping. However, a variance in eigenfrequency is noticed. During the first second, the curves coincide, then the oscillations of the simulation are slowing down compared to the measured values before finally ending a bit faster than the experimental ones. To illustrate this, the frequency of each period is shown as a function of time in Figure 14 (lower), where the mentioned difference is visible. This varying eigenfrequency of the boom is a result of the friction. As time elapses and the oscillatory motion dampens out then the stiction period where the piston and the cylinder are locked together increases until it covers the entire oscillation time. In that period, the eigenfrequency increases from the mechanical-hydraulic to the pure mechanical that can also be found in Table 6 . Because of the small deviations from the experiments and the good correlation in how the stiction influences the overall motion, the model is considered useful for a parameter study.
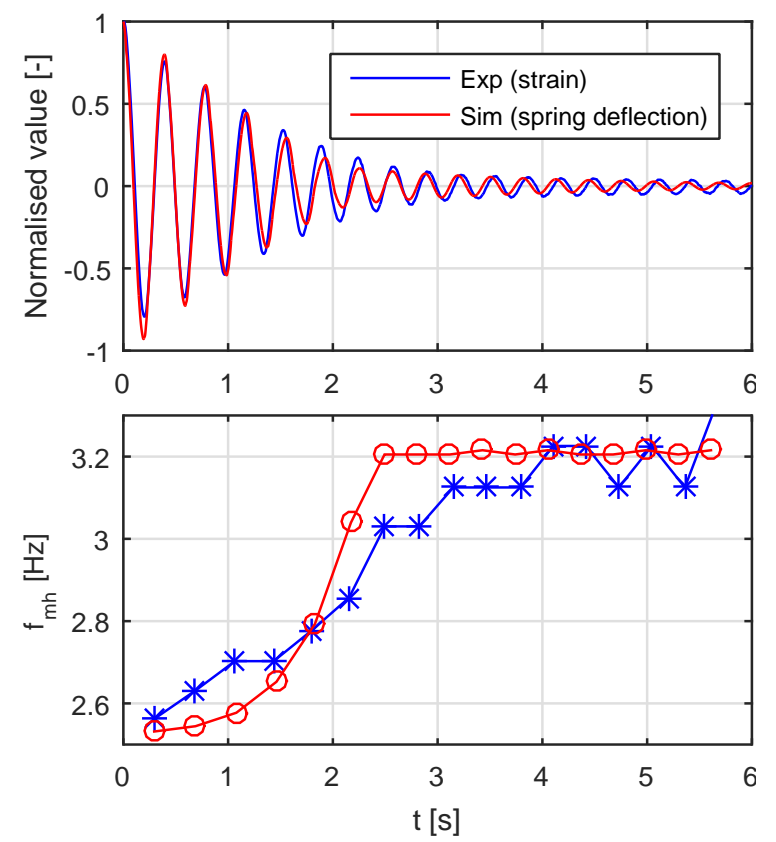

Figure 14: (Upper) Normalised values of oscillations at $x_{C}=0.10 m$. (Lower) Mechanical-hydraulic eigenfrequency $f_{m h}$, showing the frequency between each downwards zero crossing of the upper figure. 
For $x_{C}=0.25 \mathrm{~m}$ the same tendency is observed, see Figure 15. It is also noticed that the damping rate is lower in this position, due to the change in cylinder volumes.

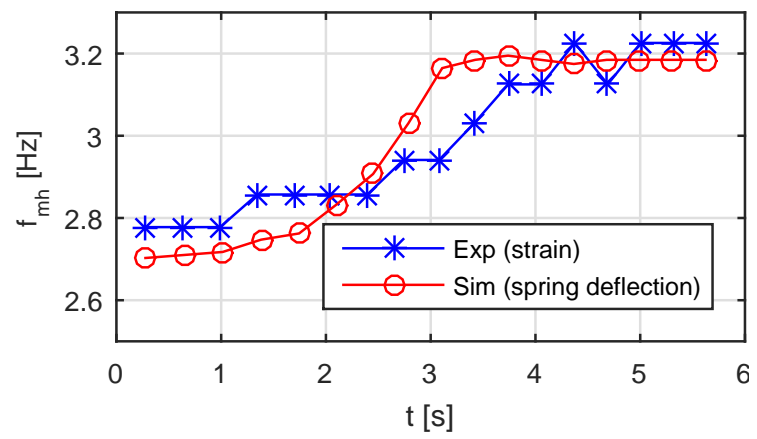

Figure 15: Mechanical-hydraulic eigenfrequency at $x_{C}=0.25 \mathrm{~m}$. The plot shows the frequency between each downwards zero crossing of a curve of the normalised values of oscillations at $x_{C}=0.25 \mathrm{~m}$.

The lower limit for the mechanical-hydraulic eigenfrequency can be found by removing the cylinder friction in the simulation. In Figure 16 this $f_{m h}^{N F}$ is shown as a function of the piston position.

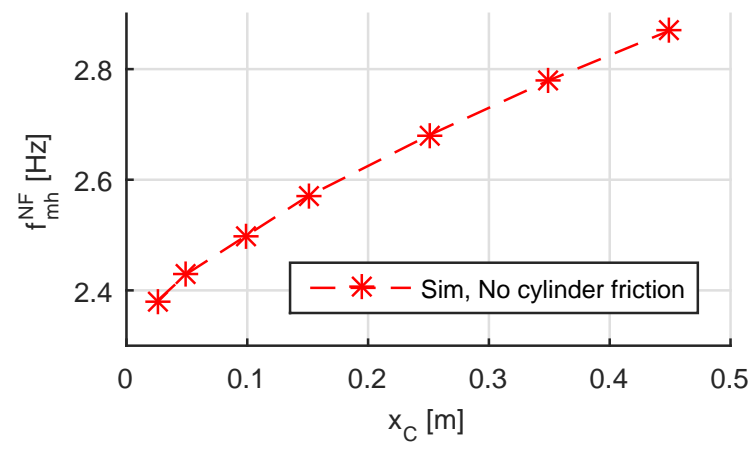

Figure 16: Mechanical-hydraulic eigenfrequency without cylinder friction, $f_{m h}^{N F}$ as a function of piston position $x_{C}$.

\subsection{Secondary circuit}

In order to be able to verify the performance of the novel concept the secondary circuit is analysed first. To check the model of the PV in equation (23), it is compared to experimental values when $p_{C}$ is plotted as a function of $u_{P V}$, see Figure 17 .

The experiment shows that an input $u_{P V}$ to the model gives the expected pressure $p_{C}$. The performance is evaluated by applying a reference step input, $p_{C}^{r e f}$, of $40 b a r$ to the secondary circuit. With

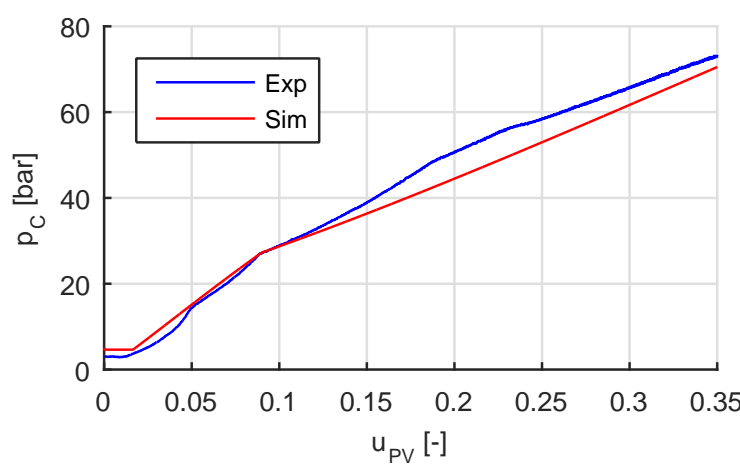

Figure 17: Verification of the PV: Pressure $p_{C}$ as a function of $u_{P V}$.

$p^{\text {min }}=5$ bar, the gains were adjusted to: $K_{P}=0.01 \frac{\mathrm{V}}{\mathrm{bar}}$ and $K_{I}=0.04 \frac{\mathrm{V}}{\mathrm{bar} \cdot \mathrm{s}}$. The results are shown in Figure 18.

The experiment consists of two parts: one where the unfiltered performance of the secondary circuit is evaluated and one where the low-pass filter is applied. The blue curve shows the reference step input. The ability of the closed-loop control system to follow this reference is shown in red and black, for the experiment and simulation. These curves are obtained without any filtration, i.e., $\tau=0 s$. The performance when applying the low-pass filter in the system is the other part. The filtered reference $p_{C}^{r e f}$ with a cut-off frequency set to $\tau=0.32 s$, is shown in cyan. The remaining curves, the green and magenta show the ability of $p_{C}$ to follow this $p_{C}^{r e f}$. In both cases, the model shows good conformity with the experiments both when stepping up and down.

\subsection{Total system}

To achieve a uniform evaluation of the total system a standard actuation of the DCV is used in the following, see Figure 19. Only situations where the cylinder is retracting are investigated.

The actuation is defined by the cycle time, $T$, a delay time to ensure static conditions, $t_{d}$, the ramp time, $t_{r}$, and the wanted steady state DCV input $u_{D C V}^{\max }$. The time parameters are equal for all tests, see Table 7 .

In the reminder of the verification section, the dashed green lines in the figures indicate when $u_{D C V}^{r e f} \neq$ 0 .

Table 7: Common parameters for all actuation.

\begin{tabular}{ccc} 
Cycle time, $T$ & Ramp time, $t_{r}$ & Time delay, $t_{d}$ \\
\hline $8 s$ & $1 s$ & $1 s$
\end{tabular}



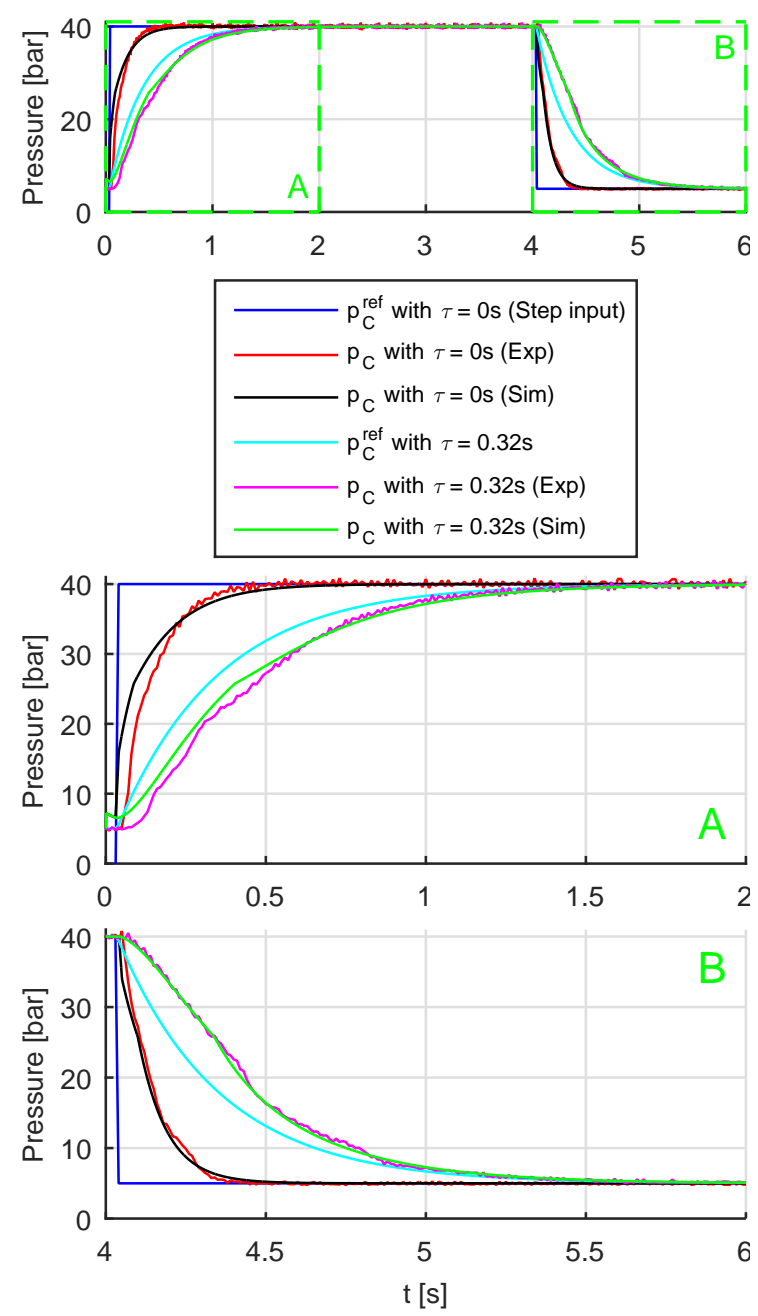

Figure 18: Comparison of the pressure responses of the secondary circuit for a reference step input, $p_{C}^{r e f}$, of 40bar. The figure comprises an overview at the top, legends, and more detailed views of the step up and the step down, respectively.

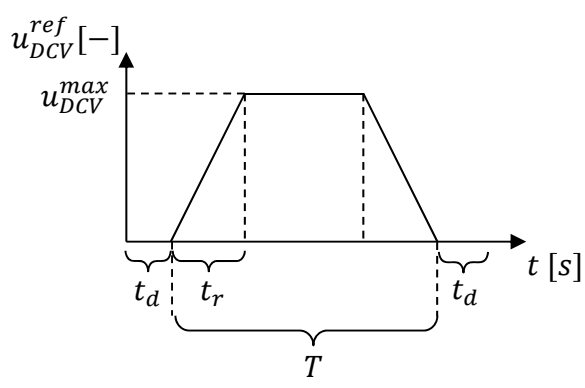

Figure 19: Work cycle - actuation function.

\subsubsection{Base circuit}

A model of the base circuit is presented to support the later verification of the novel concept. The nonlinear model of the base circuit verifies that the model of the mechanical system together with the hydraulic circuit, is providing realistic results. The system is subjected to an actuation of $u_{D C V}^{\max }=0.15$. In Figure 20 the pressures during the work cycle are shown.

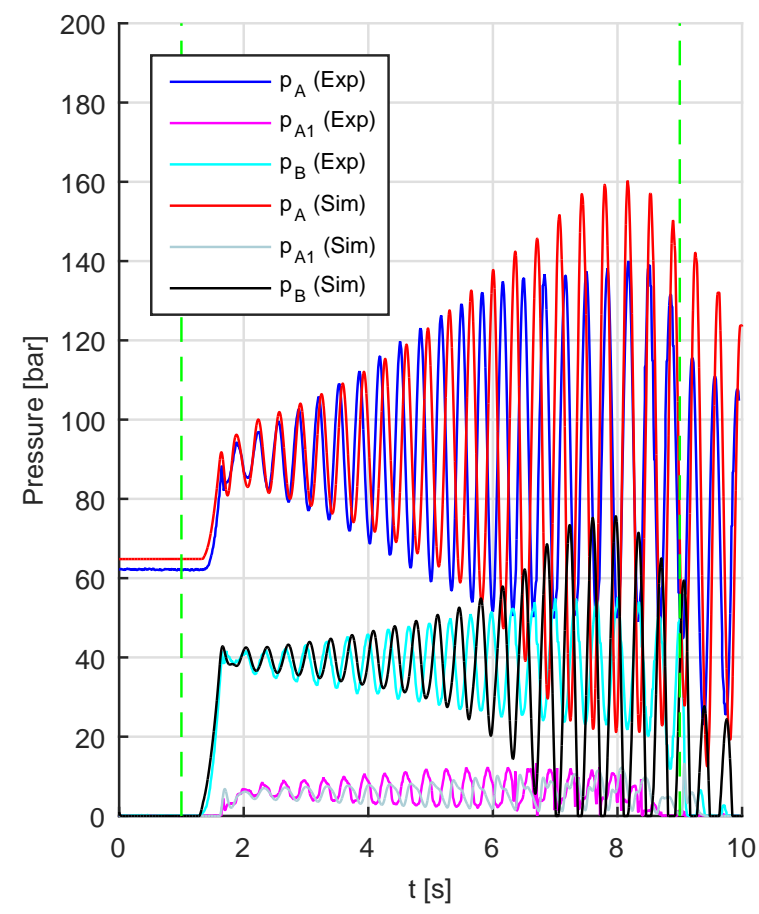

Figure 20: Comparison of pressures of the base circuit $\left(u_{D C V}^{\max }=0.15\right)$.

The markedly increased pressure amplitudes and distinct oscillations are characteristic for an unstable system, which corresponds well with the linear stability analysis in Sørensen et al. (2016). The deviations in amplitude that appear as the boom is lowered are considered acceptable due to the very violent oscillations of the physical setup that may have introduced phenomena not included in the model. A section of Figure 20 showing from $1 s$ to $5 s$, is presented in Figure 21.

The figures 20 and 21 show a satisfactory resemblance between the simulated and the measured values of the pressures. The levels of $p_{A}, p_{A 1}$ and $p_{B}$ indicate that the mechanical model and the model of the CBV resemble the real system well. The frequencies and amplitudes of the oscillations are satisfactory, although a certain drift is observed after 3-4 periods. The good correspondence of the piston velocity shown in Figure 22 indicates that the characteristics of the DCV is modelled correctly. 


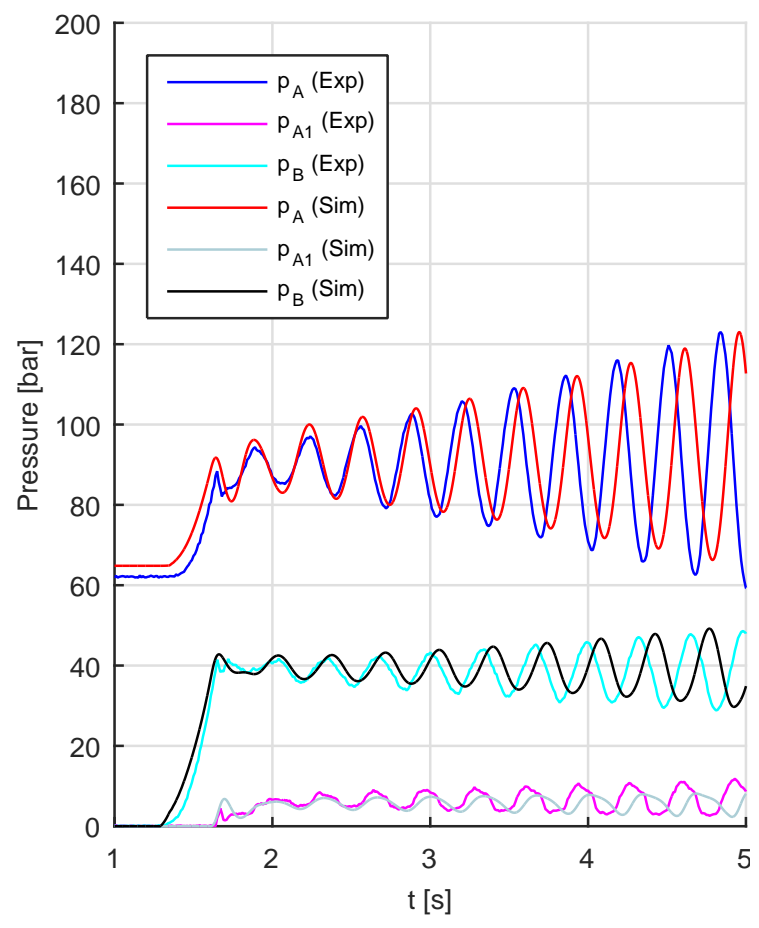

Figure 21: Zoom of pressure comparison of the base circuit $\left(u_{D C V}^{\max }=0.15\right)$ shown in Figure 20.

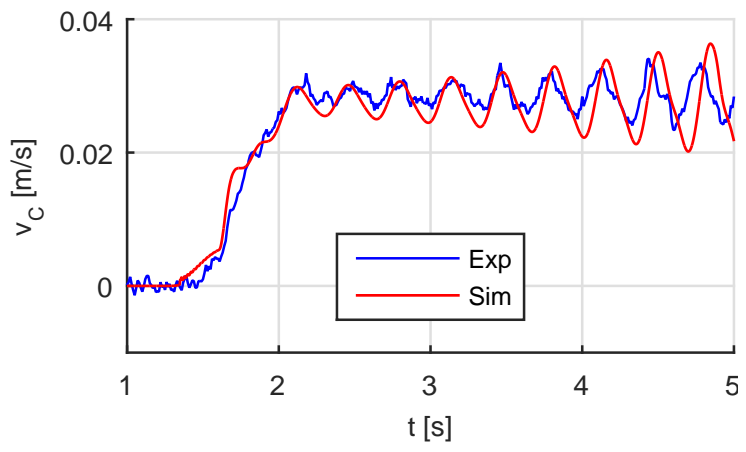

Figure 22: Comparison of piston velocity of the base circuit $\left(u_{D C V}^{\max }=0.15\right)$.
To summarise, the model of the base circuit captures the physical tendencies of the real system well. It shows good resemblance for pressure levels together with the frequencies and amplitude of the oscillations. It is concluded that the behaviour of the following parts of the nonlinear model have been verified:

- Model of mechanical system including stiffness and damping.

- Model of friction in the hydraulic cylinder.

- Model of counterbalance valve.

- Model of directional control valve.

- Model of hydraulic system including stiffness and damping.

\subsubsection{Novel concept}

The last step in the verification is to attach the secondary circuit to the base circuit and look at the novel concept. The concept is first subjected to an actuation of $u_{D C V}^{\max }=0.15$. The controller was implemented with $p^{\text {min }}=35 b a r$, and the same gains as in section 5.3. Figure 23 shows the pressures during the work cycle.

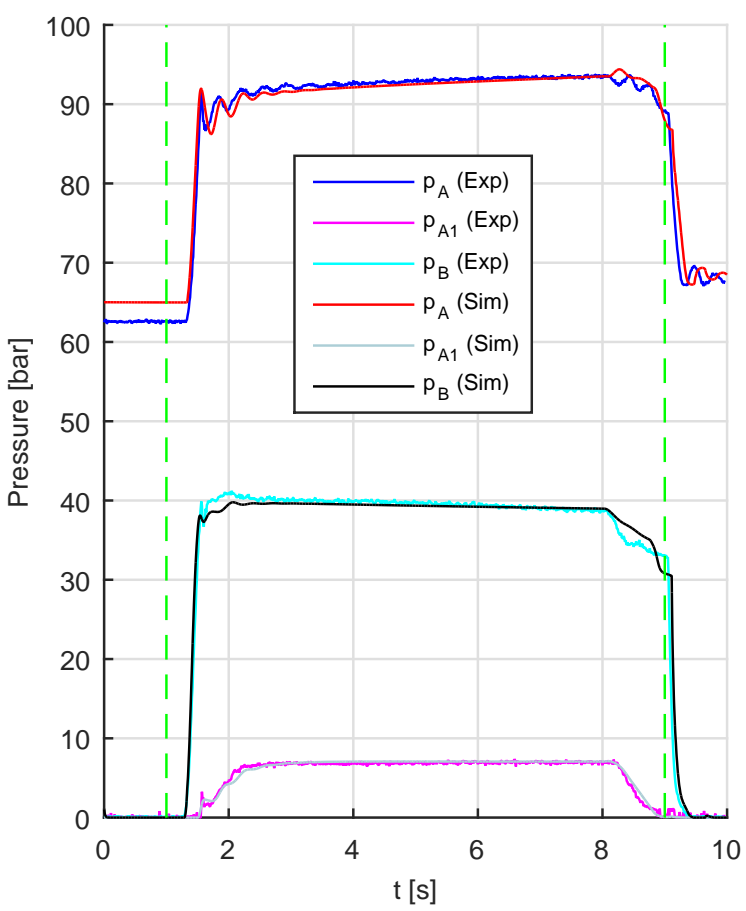

Figure 23: Comparison of pressures of the system with the concept implemented $\left(u_{D C V}^{\max }=0.15\right)$.

The simulated values correspond well with the measured ones. The oscillations in pressure $p_{A}$ at the start show good resemblance with respect to both frequency 
and amplitude. There are minor differences between model and simulation in both pressure $p_{A}$ and $p_{B}$ when the deceleration begins after $8 s$, but the general trend is followed and the pressures are deemed satisfying. The pressures of the secondary circuit are shown in Figure 24 .

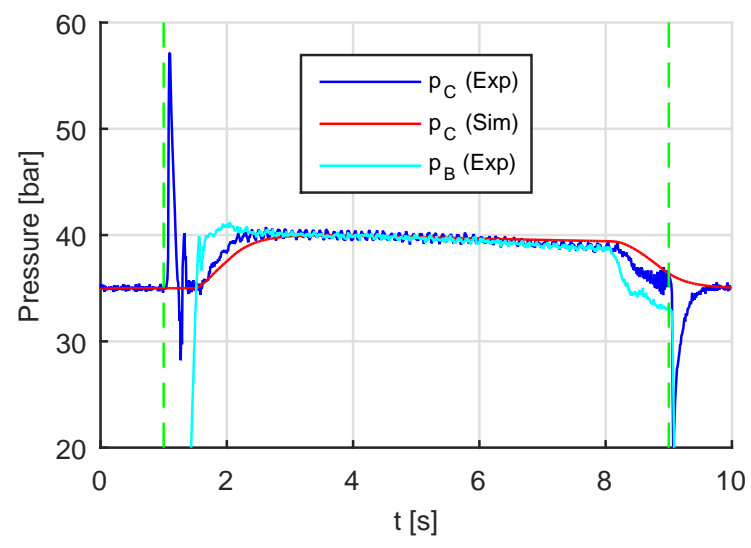

Figure 24: Pressure response of the concept's secondary circuit during work cycle $\left(u_{D C V}^{\max }=\right.$ $0.15)$.

The pressure peaks at the beginning and end of the cycle which only occur in the experiments, indicate that modelling the leakage as a fixed orifice might be an oversimplification of the LS system. A detailed analysis of this would lie outside the scope of this paper and is also considered peripheral to the more generic investigation of the concept. The measured pressure $p_{B}$ in the diagram is added to illustrate how the secondary circuit reacts to inputs from the primary circuit. Comparing the piston position and velocity in Figure 25 and Figure 26, a good resemblance is observed in both figures; for example is the small peak with negative velocity measured in the experiments at approximately $9 s$ replicated in the simulation.

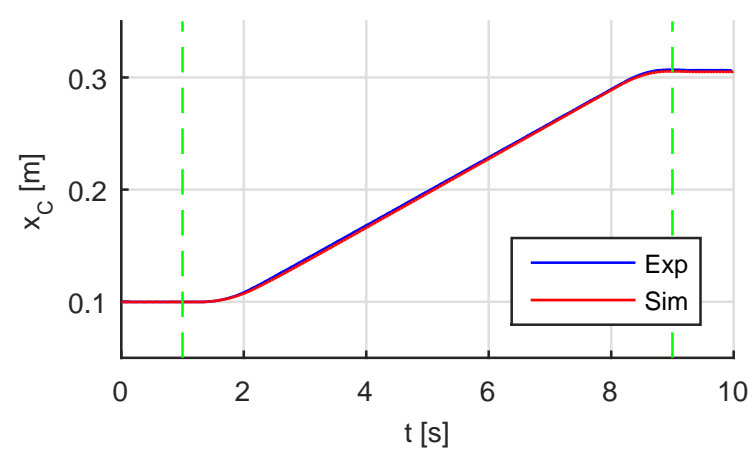

Figure 25: Comparison of piston position of the system with the concept implemented $\left(u_{D C V}^{\max }=\right.$ $0.15)$.

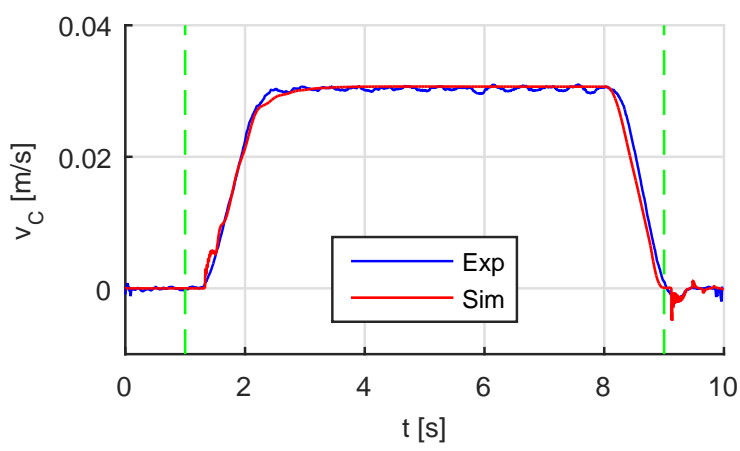

Figure 26: Comparison of piston velocity of the system with the concept implemented $\left(u_{D C V}^{\max }=\right.$ $0.15)$.

To add further depth to the verification of the model, an actuation of $u_{D C V}^{\max }=0.05$ is also analysed. Figure 27 shows the pressures during this work cycle.

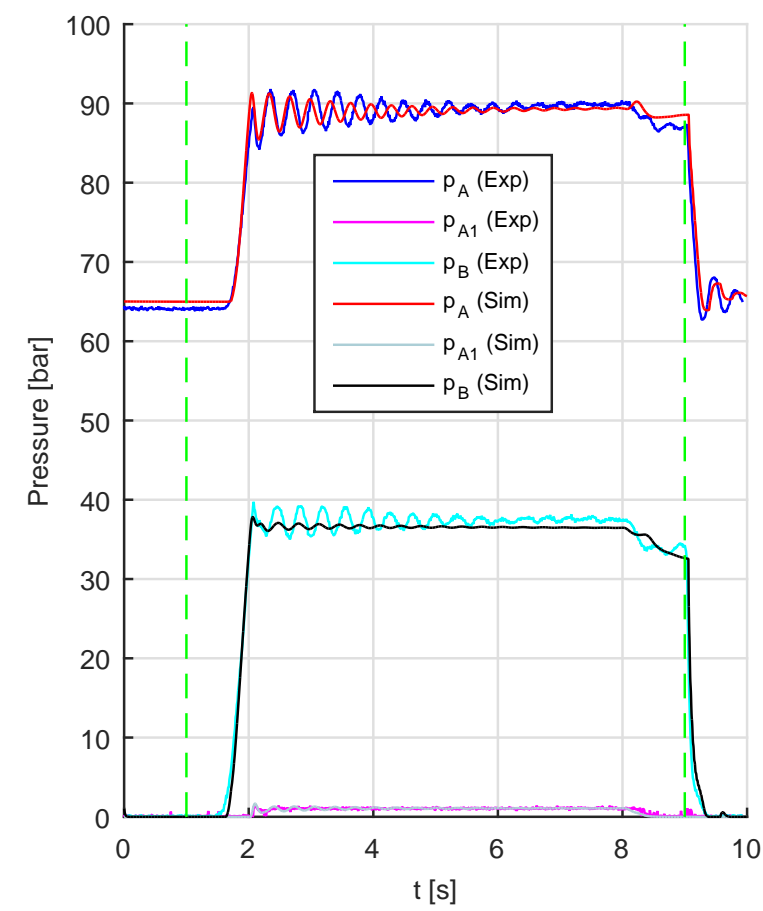

Figure 27: Comparison of pressures of the system with the concept implemented $\left(u_{D C V}^{\max }=0.05\right)$.

Also in this case the simulated values correspond well with the measured ones. However, the amplitudes are smaller in the simulation for both pressure $p_{A}$ and $p_{B}$, and this is most pronounced at the rod side of the cylinder. Figure 28 highlights this part of the pressure curve.

There is a certain discrepancy which was not seen for the base circuit, hence, the source for this deviation probably lies in the modelling of the modified 


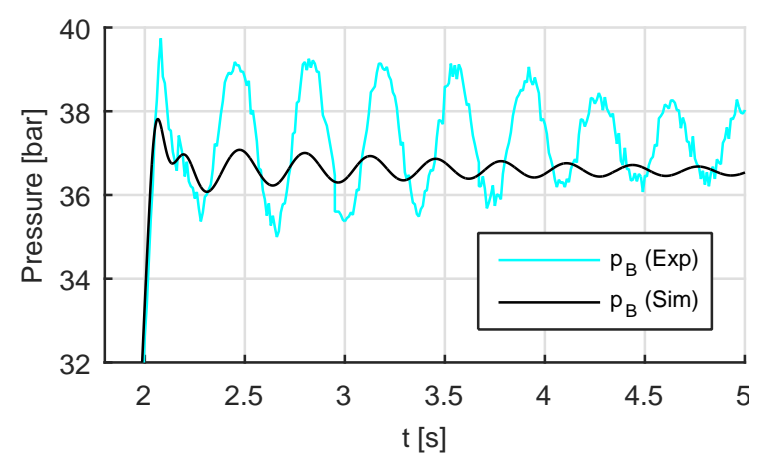

Figure 28: Zoom of part of pressure $p_{B}$ from the system with the concept implemented $\left(u_{D C V}^{\max }=\right.$ 0.05) shown in Figure 27.

DCV. As seen in Figure 24 the modifications have led to pressure fluctuations in the secondary circuit not easily accounted for and it may be the same phenomena that give more oscillations in the rod side volume of the physical system. Finally, the flow capability of the DCV is checked by comparing the steady state velocity of the piston. Experiments using the work cycle function for nine different values of $u_{D C V}^{\max }$ were conducted. The result of the tailor-made flow characteristics introduced in equation (13) can be seen in Figure 29, clearly indicating that the deviations are virtually eliminated.

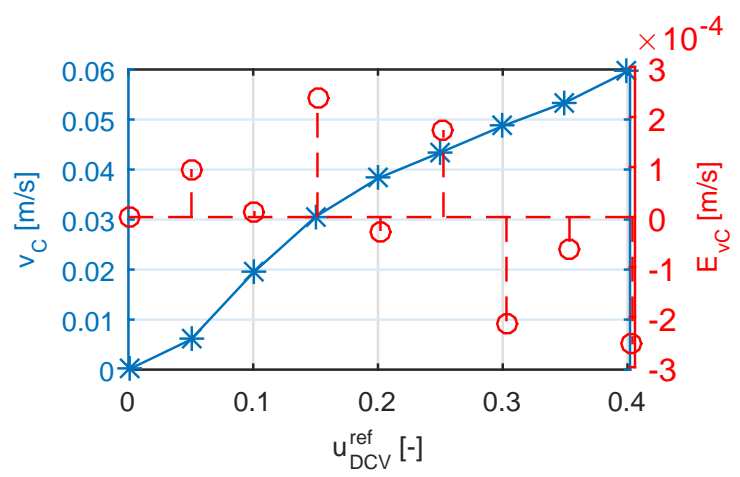

Figure 29: Simulated steady state piston velocities for different valve openings for the system with the concept implemented. The velocity error $E_{v C}=v_{C}(\operatorname{Exp})-v_{C}($ Sim $)$.

A nonlinear simulation model has been developed for the novel concept applied as actuation for a specific cylinder-boom mechanism. In general, the model corresponds well both steady state and dynamically with measured data and it is further validated by simulations and experiments conducted using a base circuit as actuation for the same mechanism.

\section{Parameter study}

The nonlinear model is utilised to investigate which parameters yield the largest influence on the stability of the novel concept. A linear stability analysis has indicated that small openings of the directional control valve result in stability issues (Sørensen et al., 2016). That linear analysis was, however, based on a simplified system and therefore a parameter study is conducted here with a view to investigate the oscillatory behaviour of the nonlinear system. Since stability is associated with linear systems the term is adapted to the current study based on how the pressure amplitudes develop after the ramp up is conducted, i.e. $u_{D C V}^{r e f}=u_{D C V}^{\max }$. Increasing amplitudes are clearly characteristics of a highly oscillatory system in this context and are simply referred to as unstable. The way the concept is working with the secondary circuit separated from the primary circuit by a low-pass filter lowers the performance requirements to the components in the secondary circuit, hence its influence on the stability of the system is limited. The cylinder friction does not yield much effect on the stability either. Simulations show that the parameters most influential on the systems stability are the stiffness of the mechanical structure and the pilot area ratio of the CBV. In Figure 30 the blue curve shows the minimum $u_{D C V}^{r e f}$ yielding a stable system as a function of $f_{m h}^{N F}$ when the starting position of the piston is $x_{C}=0.10 \mathrm{~m}$. The system becomes increasingly oscillatory when the stiffness and hence the eigenfrequency increases. Also notice that for $f_{m h}^{N F}<2.2 \mathrm{~Hz}$ the simulation becomes stable for all valve openings.

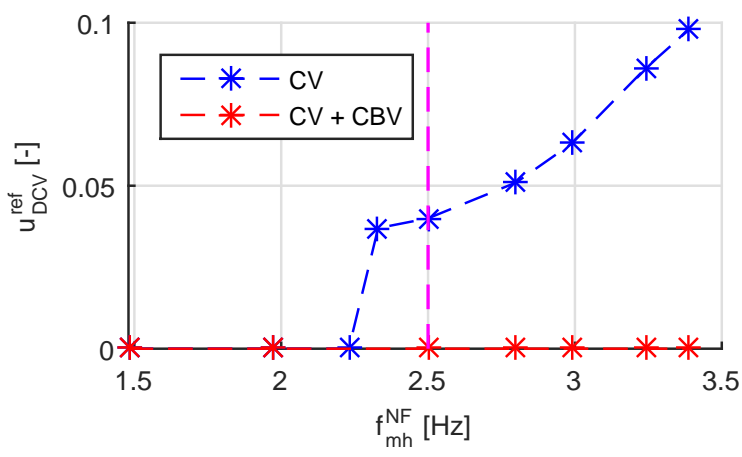

Figure 30: Stability of the nonlinear model of the novel concept; both connected to $\mathrm{CV}$ and $\mathrm{CV}+\mathrm{CBV}$. The diagram shows the minimum $u_{D C V}^{r e f}$ that yields a stable system as a function of $f_{m h}^{N F}$ for $\alpha_{P}=3$. The dashed magenta line is the value of the real system.

Curves for a varying pilot area ratio of the CBV are shown in Figure 31. Stability is improved by lowering 
the pilot area ratio. This of course happens at the expense of a more pronounced pressure-load dependency in the cylinder chambers. The blue curve also indicates that for pilot ratios $\alpha_{P}<1.9$ stability can be ensured in all cases.

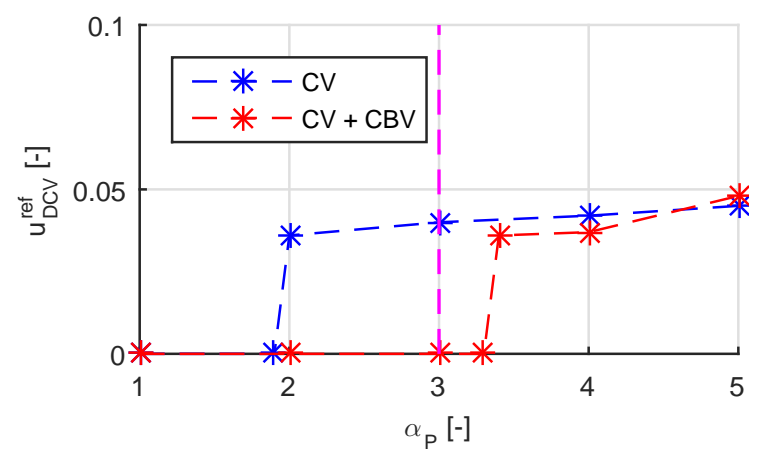

Figure 31: Stability of the nonlinear model of the novel concept; both connected to $\mathrm{CV}$ and $\mathrm{CV}+\mathrm{CBV}$. The diagram shows the minimum $u_{D C V}^{r e f}$ that yields a stable system as a function of $\alpha_{P}$ for $f_{m h}^{N F}=2.5 H z$. The dashed magenta line is the value of the real system.

In both Figure 30 and Figure 31 the curves for $\mathrm{CV}$ indicate that for the system in the lab $\left(f_{m h}^{N F}=2.5 \mathrm{~Hz}\right.$ and $\alpha_{P}=3$ ) instability occurs for $u_{D C V}^{r e f}$ below approximately 0.04 , for this specific system. In some applications, the possible risk of oscillations for small valve openings might be unacceptable. As mentioned in the presentation of the concept the solution also encompasses a version where the secondary circuit besides being connected to the $\mathrm{CV}$ also controls the opening of the $\mathrm{CBV}(\mathrm{CV}+\mathrm{CBV})$. The hydraulic diagram of this circuit is shown in Figure 32.

The results of this change in the hydraulic circuit are shown in red in Figure 30 and Figure 31. If $\alpha_{P}=3$, this solution does not experience instability for any value of $f_{m h}^{N F}$. When varying the pilot area ratio, a clear improvement can be observed. The stability threshold increases and the system is now stable in the configuration of the real setup $\left(\alpha_{P}=3\right)$. This proves that the concept is able to stabilise the experimental setup for all openings of the directional control valve. Controlling the opening area of the $\mathrm{CBV}$ via a separate pressure source can be regarded problematic from a reliability point of view in some applications, since the CBV provides different safety functions, among them load holding at hose/pump failure. Therefore, the solution indicated in Figure 32 should only be considered if instability cannot be overcome by lowering the pilot area ratio.

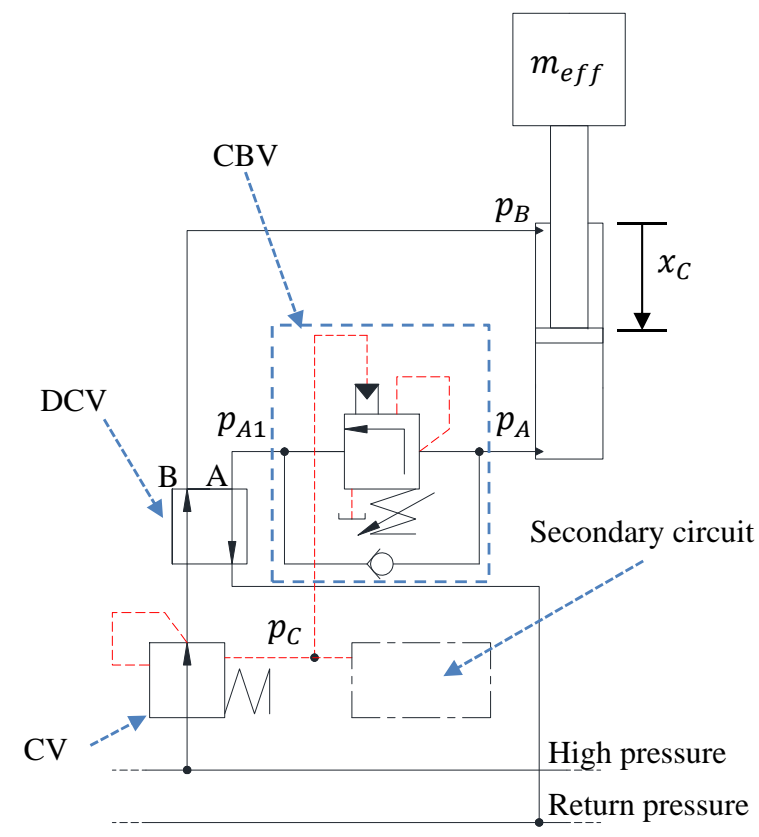

Figure 32: Hydraulic diagram of novel concept. Both the $\mathrm{CBV}$ and the $\mathrm{CV}$ are connected to the secondary circuit.

\section{Conclusions}

The authors have previously presented a novel concept capable of suppressing oscillations in hydraulic systems containing a CBV and a pressure compensated directional control valve (DCV), see Sørensen et al. (2016). The concept utilizes a secondary circuit where a lowpass filtered value of the load pressure is generated and fed back to the compensator of the flow supply valve. This paper has focused on a further investigation of this concept and limitations hereof. A nonlinear dynamic model has been developed and experimentally verified on a cylinder actuated single boom mechanism. The commercial simulation tool SimulationX has been used as platform for the modeling. The mechanical system is modelled as a multi-body system using the finite segment flexibility method. The hydraulic circuit including the main control components have been modelled using a combination of liquid volumes, variable orifices and $1^{\text {st }}$ and $2^{\text {nd }}$ order transfer functions to capture valve dynamics. The eigenfrequencies of both the mechanical and the combined mechanical-hydraulic system and the secondary circuit were investigated and validated separately - before being combined to a model of the entire system. In order to strengthen the verification, a model of the same mechanical-hydraulic system actuated by means of a standard base circuit was also investigated both experimentally and numerically. This ensured that the devel- 
oped models of the mechanical system and the counterbalance valve (CBV) could be verified for two different setups. The full nonlinear model of the mechanicalhydraulic system actuated by the concept was in general, in good accordance with measurements. During the modelling of the mechanical-hydraulic system in this paper the following areas showed themselves to be of high importance:

- Flexibility of the mechanical system.

- Friction in the hydraulic cylinder.

- Continuous opening of the CBV.

- Proper characteristics of the DCV.

Since the main feature of the concept is its ability to suppress oscillations, the developed model was used for a parameter study with emphasis on instability. The model confirmed the results from the linear analysis in Sørensen et al. (2016), that there is an elevated risk for instability at small DCV openings. The model shows that an improved system stability can be obtained by either reducing the eigenfrequency of the mechanicalhydraulic system or lowering the pilot area ratio of the CBV. Finally, the model showed the improved stability characteristics of another version of the concept where also the CBV pilot port is connected to the low pass filtered load pressure. This version would normally be considered less desirable from a reliability point of view because the basic safety features of the CBV are controlled electronically, however, the simulations indicate that it could be an alternative for systems that cannot be stabilised otherwise.

\section{Acknowledgments}

The work is funded by the Norwegian Ministry of Education \& Research and National Oilwell Varco.

\section{References}

Bak, M. K. and Hansen, M. R. Analysis of offshore knuckle boom crane - part one: Modeling and parameter identification. Modeling, Identification and Control, 2013. 34(4):157-174. doi:10.4173/mic.2013.4.1.

Cristofori, D., Vacca, A., and Ariyur, K. A novel pressure-feedback based adaptive control method to damp instabilities in hydraulic machines. SAE Int. J. Commer. Veh, 2012. 5(2):586-596. doi:10.4271/2012-01-2035.
Handroos, H., Halme, J., and Vilenius, M. Steady-state and dynamic properties of counter balance valves. In Proc. 3rd Scandinavian International Conference on Fluid Power. Linköping, Sweden, 1993.

Hansen, M. R. and Andersen, T. O. A design procedure for actuator control systems using optimization methods. In Proc. 7th Scandinavian International Conference on Fluid Power. Linköping, Sweden, 2001.

Hansen, M. R. and Andersen, T. O. Controlling a negative loaded hydraulic cylinder using pressure feedback. In Proc. 29th IASTED International Conference on Modelling, Identification and Control. Innsbruck, Austria, 2010. doi:10.2316/P.2010.675-116.

Hansen, M. R. and Sørensen, J. K. Improvements in the control of hydraulic actuators (pending). 2015.

Huston, R. and Wang, Y. Flexibility effects in multibody systems. In M. Pereira and J. Ambrsio, editors, Computer-Aided Analysis of Rigid and Flexible Mechanical Systems, pages 351-376. Kluwer Academic Publishers, Dordrecht, NL, 1994.

Miyakawa, S. Stability of a hydraulic circuit with a counter-balance valve. Bulletin of the JSME, 1978. 21(162):1750-1756.

Nordhammer, P., Bak, M. K., and Hansen, M. R. A method for reliable motion control of pressure compensated hydraulic actuation with counterbalance valves. In Proc. 12th International Conference on Control, automation and systems. Jeju Island, Korea, 2012.

Persson, T., Krus, P., and Palmberg, J.-O. The dynamic properties of over-center valves in mobile systems. In Proc. 2nd International Conference on Fluid Power Transmission and Control. Hangzhou, China, 1989.

Ritelli, G. and Vacca, A. A general auto-tuning method for active vibration damping of mobile hydraulic machines. In Proc. 8th FPNI Ph.D. Symposium on Fluid Power. Lappeenranta, Finland, 2014.

Sørensen, J. K., Hansen, M. R., and Ebbesen, M. K. Boom motion control using pressure control valve. In Proc. 8th FPNI Ph.D. Symposium on Fluid Power. Lappeenranta, Finland, 2014.

Sørensen, J. K., Hansen, M. R., and Ebbesen, M. K. Load independent velocity control on boom motion using pressure control valve. In Proc. 14th Scandinavian International Conference on Fluid Power. Tampere, Finland, 2015. 
Sørensen, J. K., Hansen, M. R., and Ebbesen, M. K. Novel concept for stabilizing a hydraulic circuit containing counterbalance valve and pressure compensated flow supply. International Journal of Fluid Power, 2016. 17(3):153-162. doi:10.1080/14399776.2016.1172446.

Zähe, B. Stability of load holding circuits with counterbalance valve. In Proc. 8th International Bath Fluid Power Workshop. Bath, UK, 1995. 